\title{
Activation of vagal afferents by veratrine induces antinociception
}

\author{
ALAN RANDICH, THOMAS A. SIMPSON, PHILIP A. HANGER, \\ and ROBYN L. FISHER \\ The University of Iowa, Iowa City, Iowa
}

\begin{abstract}
Experiment 1 showed that intravenous administration of veratrine resulted in inhibition of the tail-flick reflex, bradycardia, and hypotension. These antinociceptive and cardiovascular actions of veratrine were unaffected by pretreatment with iv administration of $.5 \mathrm{mg} / \mathrm{kg}$ of the opioidreceptor antagonist naloxone. Experiment 2 demonstrated that veratrine-induced inhibition of the tail-flick reflex was eliminated by bilateral cervical vagotomy in an anesthetized rat preparation, but was unaffected by either a unilateral right or left vagotomy in a conscious rat preparation. Experiment 3 showed that intravenous administration of veratrine resulted in inhibition of the paw-lick response to painful thermal heat and that this antinociceptive action of veratrine was unaffected by pretreatment with systemic administration of $20 \mathrm{mg} / \mathrm{kg}$ naloxone. These experiments show that veratrine-induced antinociception is mediated by peripheral activation of vagal afferents.
\end{abstract}

Several lines of evidence suggest that vagal afferents arising from the cardiopulmonary region are linked to systems involved in the processing of painful stimuli. Electrophysiological evidence supporting this view derives from studies showing that electrical stimulation of vagal afferents attenuates the firing of low-threshold, wide dynamic range and high threshold spinothalamic neurons projecting from laminae I, II, V, and VII of the spinal dorsal horn (Ammons, Blair, \& Foreman, 1983a, 1983b; Theis \& Foreman, 1981). Behavioral evidence supporting the view of a vagal influence on pain perception derives from recent work showing that physiological activation of cardiopulmonary mechanoreceptors by volume expansion results in long-lasting inhibition of the tail-flick reflex evoked by painful radiant heat in the rat, and this effect is attenuated in rats with right cervical vagotomy (Maixner \& Randich, 1984). Perhaps of greater significance, intravenous administration of [D-Ala $\left.{ }^{2}\right]-$ methionine enkephalinamide (DALA) induces inhibition of the tail-flick reflex to painful heat in the rat. This antinociceptive action of DALA is eliminated in rats receiving either pretreatment with $1 \mathrm{mg} / \mathrm{kg}$ of naloxone or bilateral cervical vagotomy, but prior unilateral right vagotomy or bilateral sinoaortic deafferentation (SAD) has no effect on the antinociception (Randich \& Maixner, 1984a). Furthermore, resection of the right cervical vagal nerve trunk both reverses the opioid-mediated antinociception manifested by the spontaneously hypertensive rat (SHR) in the hot-plate assay of pain sensitivity (Maixner, Touw, Brody, Gebhart, \& Long, 1982), and attenuates stress-induced analgesia (SIA) resulting from

\footnotetext{
This research was supported by a grant from N.I.H. (NS 18341) to A. Randich. We thank NIDA for their generous contribution of naloxone and R. T. Ross for comments on an earlier version of this manuscript.

The authors' mailing address is: Department of Psychology, University of Iowa, Iowa City, IA 52242.
}

exposure to $30 \mathrm{~min}$ of intermittent footshock (Maixner \& Randich, 1984). Collectively, these findings have been interpreted to support the more general view that peripheral and central systems involved in cardiovascular regulation are physiologically linked to systems involved in pain perception to form a functional network governing the elaboration of adaptive responses to physical and psychological stressors (for a review, see Randich \& Maixner, 1984b).

The present series of experiments provide additional evidence in support of this view by assessing antinociceptive actions of the compound veratrine. Veratrine is a combination of veratrum alkaloids that nonselectively stimulates cardiac mechanoreceptors and chemosensitive receptors with associated vagal afferents (Bishop, Malliani, \& Thoren, 1984; Kupchan \& Flacke, 1967), typically resulting in both reflex bradycardia and hypotension: the Bezold-Jarisch reflex (Thoren, 1979). According to the view of Randich and Maixner (1984b), administration of veratrine is likely to result in antinociception and be mediated by activation of peripheral receptors with associated vagal afferents. Therefore, the purpose of these experiments is fourfold: (1) to show that iv administration of veratrine induces inhibition of the tail-flick reflex to painful radiant heat, (2) to provide a preliminary assessment of the possible involvement of endogenous opioids, (3) to determine whether veratrine induces antinociception by activating vagal afferents at the peripheral level or by acting directly on the CNS, and (4) to determine if veratrine also induces inhibition of the paw-lick response to painful thermal stimulation.

\section{EXPERIMENT 1}

The purpose of Experiment 1 was to assess the antinociceptive properties of an iv bolus infusion of vera- 
trine. This was accomplished using the tail-flick reflex elicited by radiant heat as an index of pain sensitivity. The antinociceptive actions of veratrine were also characterized following prior administration of the opioid-receptor antagonist naloxone.

\section{Method}

Subjects. Five male Sprague-Dawley rats obtained from Hormone Assay Laboratories in Chicago served as subjects. The rats were individually housed in wire-mesh cages under a 12:12-h light:dark cycle. Food and water were available on an ad-lib basis.

Apparatus. Nociceptive responses were measured with a tailflick apparatus. The radiant heat stimulus was provided by a $500-\mathrm{W}$ projector bulb housed in a metal casing and focused on the rat's tail through a small opening in the metal housing. Onset and termination of each trial were controlled automatically by a digital timer. The intensity of the radiant heat stimulus, which was constant for all subjects, was intended to produce a tail-flick response of approximately 3-4 sec in a normal rat.

Arterial blood pressure and heart rate were recorded on a Beckman $\mathrm{R} 11 \mathrm{~A}$ rectilinear dynagraph from the signal provided by a Century pressure transducer.

Surgical Techniques. Each rat was anesthetized with pentobarbital sodium $(50 \mathrm{mg} / \mathrm{kg})$. An incision was made in the pectoral region $1 \mathrm{~cm}$ to the right of the midventral line and extending $2 \mathrm{~cm}$ craniad from the pectoralis. The external jugular vein was exposed at its junction with the right subclavian vein, and the connective tissue surrounding the area was cleared. A cannula (Silastic) was inserted caudad through the brachiocephalic vein approximately $4 \mathrm{~cm}$. The cannula was anchored to adjacent tissue.

A $3-\mathrm{cm}$ midventral incision was then made in the cervical region. Dissection continued until the underlying sternocleidomastoid group was exposed. The left sternomastoid was reflected laterally, and the deeper omohyoid was cut at right angles to its fiber direction. The left common carotid artery was then separated from the surrounding tissues and nerves. A cannula was advanced caudad 3$4 \mathrm{~cm}$ and anchored to adjacent muscles (Microline).

The arterial and venous cannulae were then drawn subcutaneously around the neck and exited through a dorsal incision. The cannulae were anchored to the neck and flushed with a saline-heparin solution.

Testing. All rats were tested $24 \mathrm{~h}$ after surgery. Each rat was placed in a Plexiglas restraining tube, and the cannulae were connected. Tail-flick trials were then administered until baseline latencies were between 3-4 sec and remained stable from trial to trial. Following stabilization of the tail-flick response, each rat received successive bolus iv infusions of isotonic saline and 5, 50, and $100 \mu \mathrm{g} / \mathrm{kg}$ of veratrine $\mathrm{HCl}$ (Sigma Co.). Tail-flick trials were administered $0.25,1,2$, and $3 \mathrm{~min}$ after each drug dose. Veratrine was dissolved in isotonic saline. All drugs and saline vehicle were infused in a volume of $1 \mathrm{ml} / \mathrm{kg}$ at a rate of approximately $200 \mu \mathrm{l} / \mathrm{sec}$. Each drug or saline infusion was followed by a $400-\mu$ l saline flush. Arterial blood pressure and heart rate were continuously recorded. Approximately $10 \mathrm{~min}$ intervened between administration of each successive drug dose, but trials were never initiated unless baseline tail-flick latencies had returned to normal. A maximum latency of $10 \mathrm{sec}$ was arbitrarily used to prevent tissue damage to the tail.

On the following day, each rat was again tested in a similar manner, except that saline, $500 \mu \mathrm{g} / \mathrm{kg}$ of naloxone $\mathrm{HCl}$, and $100 \mu \mathrm{g} / \mathrm{kg}$ of veratrine were successively administered iv. This dose of naloxone was chosen because it blocks morphine analgesia and enkephalinamide analgesia in our laboratory.

Data analysis. Tail-flick latencies were converted to tail-flick indices by the equation (trial latency - baseline latency)/(10 seconds - baseline latency) $\times 100$. In addition to the cardiovascular measures obtained at the 0.25-, 1-, 2-, and 3-min trial points, the largest cardiovascular responses manifested prior to the $0.25-\mathrm{min}$ trial point were recorded and defined as the "peak" responses. Tailflick indices, percentage change from baseline blood pressure, and percentage change from baseline heart rate (where peak responses are also included for cardiovascular measures) were subjected to analysis of variance. In each analysis in which the overall null hypothesis was rejected, Scheffé post hoc comparisons were performed on the means. Alpha was set at 0.05 .

\section{Results}

The outcomes of the first experiment involving administration of veratrine are presented in Figure 1. The top panel shows the mean tail-flick indices evoked by each drug dose across the four test trial time points. Mean baseline tail-flick latencies obtained immediately prior to the saline, 5-, 50-, and $100-\mu \mathrm{g} / \mathrm{kg}$ test trials were $3.17,2.72$, 2.81 , and $3.04 \mathrm{sec}$, respectively. In general, veratrine induced antinociception at both the $50-$ and $100-\mu \mathrm{g} / \mathrm{kg}$ doses. This was confirmed by an ANOVA of tail-flick indices, indicating a significant effect of drug dose $[F(3,12)=$ $46.08]$ and a significant effect of time $[\mathrm{F}(3,12)=4.03]$, but no significant drug dose $\times$ time interaction. Post hoc comparisons performed on the drug dose means obtained by collapsing across the time dimension indicated that both the $50-$ and $100-\mu \mathrm{g} / \mathrm{kg}$ dose means differed significantly from the saline mean (Fs of 4.05 and 35.84, respectively). However, the $5-\mu \mathrm{g} / \mathrm{kg}$ dose mean did not differ from that of saline.

The middle panel of Figure 1 presents mean arterial blood pressures evoked by the various doses of veratrine expressed as percentage change from baseline values. Mean baseline arterial blood pressures obtained immediately prior to the saline, $5-, 50-$ and $100-\mu \mathrm{g} / \mathrm{kg}$ test trials were $113,118,118$, and $112 \mathrm{mmHg}$, respectively. In general, veratrine induced hypotension only at the $100-\mu \mathrm{g} / \mathrm{kg}$ dose. This was confirmed by an ANOVA of arterial blood pressures indicating a significant effect of drug dose $[F(3,12)=8.86]$ and a significant effect of time $[F(4,16)=11.20]$, but no drug dose $\times$ time interaction . Post hoc comparisons indicated that only the mean arterial blood pressure evoked by the $100-\mu \mathrm{g} / \mathrm{kg}$ dose of veratrine was significantly lower than that evoked by saline $(\mathrm{F}=5.82)$.

The data for heart rate are presented in the bottom panel of Figure 1. Mean baseline heart rates obtained immediately prior to the saline, $5-, 50-$, and $100-\mu \mathrm{g} / \mathrm{kg}$ test trials were $528,544,532$, and $530 \mathrm{bpm}$, respectively. It should be noted that these very high heart rate values reflect the repeated application of the painful radiant heat stimulus necessary to establish consistent, short-latency baseline tail-flick responses. In general, veratrine elicited bradycardia at the two larger dosage levels. This was confirmed by an ANOVA of heart rates indicating a significant effect of drug dose $[\mathrm{F}(3,12)=11.39]$, a significant effect of time $[F(4,16)=23.01]$, and a significant drug dose $\times$ time interaction $[F(12,48)=6.18]$. Since the interaction was significant, individual ANOVAs were performed at each test-trial time point with appropriate follow-up comparisons. In general, these post hoc analyses indicated that only the bradycardia evoked by the 


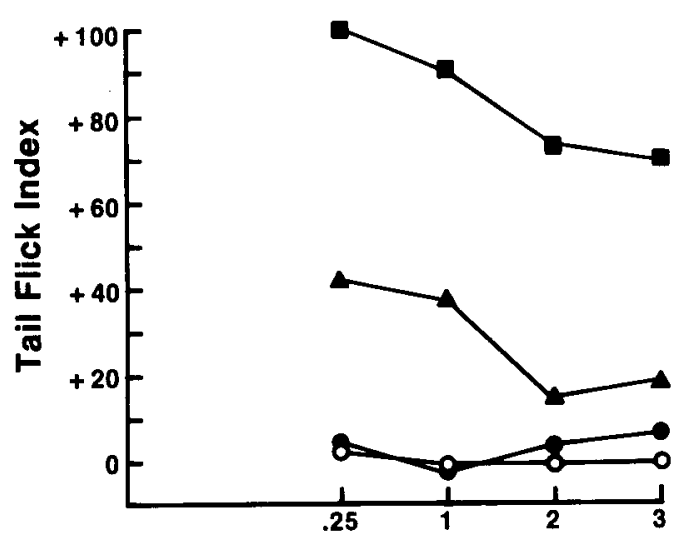

not affect the capacity of the $100-\mu \mathrm{g} / \mathrm{kg}$ dose of veratrine to induce antinociception. An ANOVA supported these impressions by indicating a significant effect of drug dose $[F(2,8)=86.75]$, but no significant effect of either time or the drug dose $\times$ time interaction. More importantly, a within-subject comparison of tail-flick indices produced by $100 \mu \mathrm{g} / \mathrm{kg}$ of veratrine in either the absence of naloxone (see Figure 1) or the presence of naloxone (see Figure 2) indicated no significant differences. This anal-
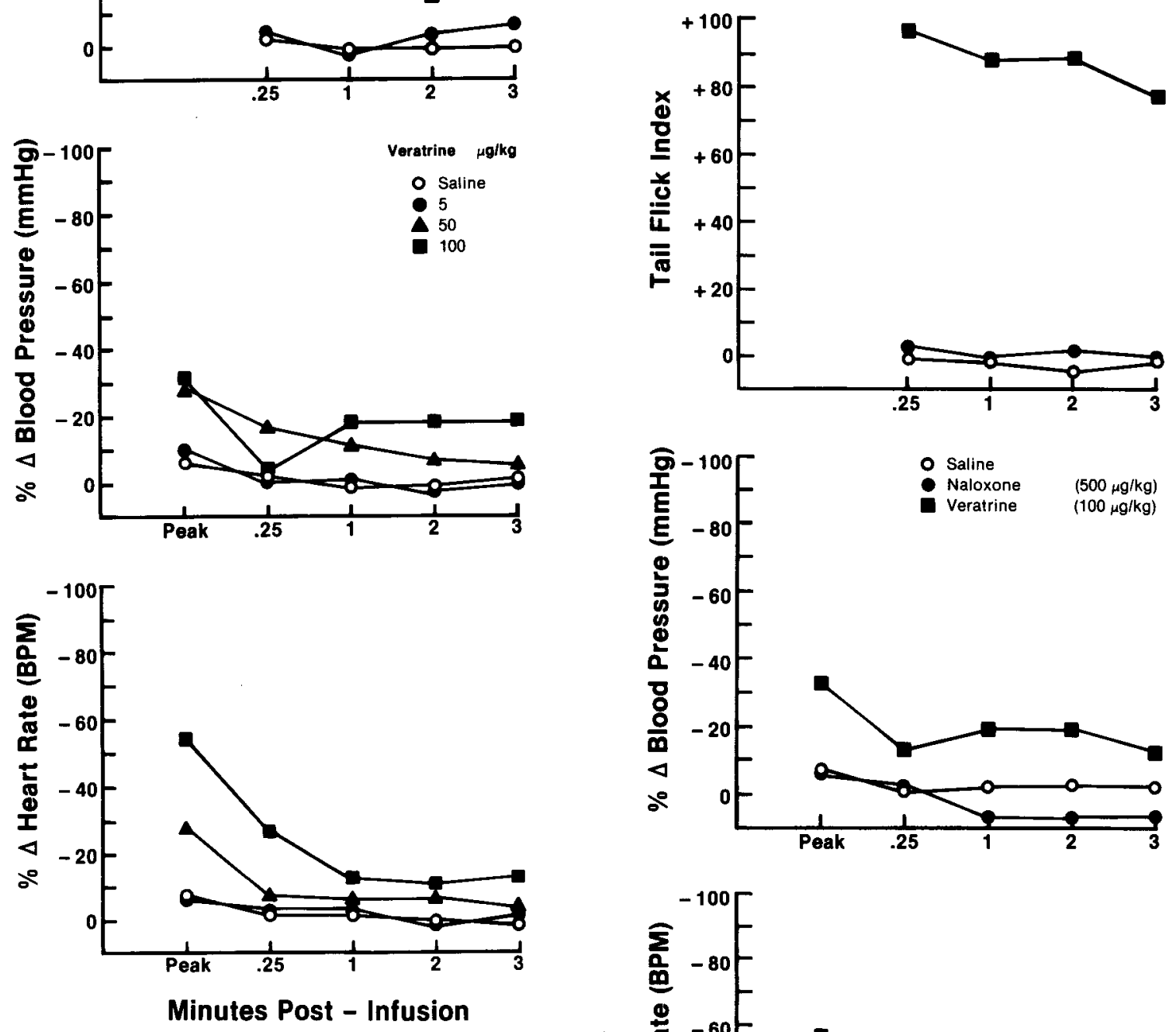

Figure 1. Mean tail-flick index, mean percent change in arterial blood pressure, and mean percent change in heart rate as a function of saline or veratrine administration.

$100-\mu \mathrm{g} / \mathrm{kg}$ dose of veratrine differed significantly from saline at the peak, 0.25-, 1-, and 3-min time points $[\mathrm{Fs}=6.81,4.72,3.87$, and 5.69 , respectively $]$.

The outcomes of the naloxone manipulation are presented in Figure 2. Mean baseline tail-flick latencies obtained immediately prior to the saline, naloxone alone, and $100 \mu \mathrm{g} / \mathrm{kg}$ of veratrine test trials were $3.42,3.04$, and $3.15 \mathrm{sec}$, respectively. The top panel of Figure 2 indicates that pretreatment with $0.5 \mathrm{mg} / \mathrm{kg}$ of naloxone did
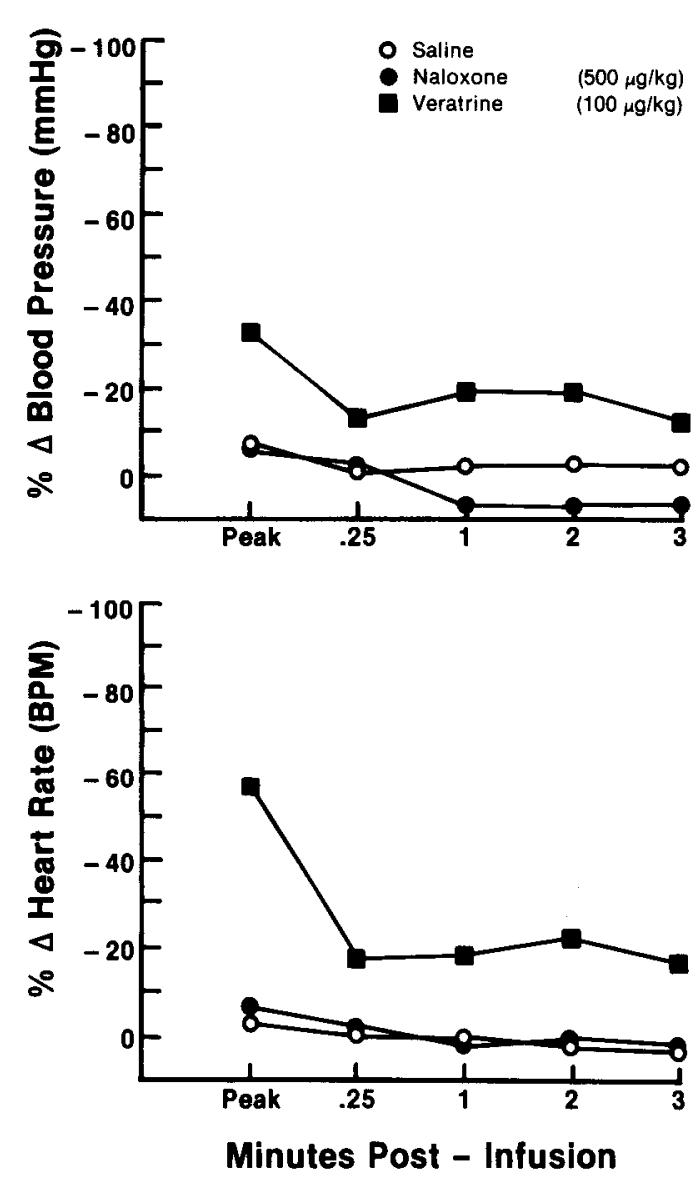

Figure 2. Mean tail-flick index, mean percent change in arterial blood pressure, and mean percent change in heart rate as a function of saline, naloxone alone, or naloxone and veratrine combined. 
ysis also indicates that it is unlikely that cumulative drug effects resulting from successive administration of veratrine doses, that is, 5,50 , and $100 \mu \mathrm{g} / \mathrm{kg}$, contributed to the nociceptive functions shown in Figure 1.

The arterial blood pressure and heart rate changes produced by administration of $100 \mu \mathrm{g} / \mathrm{kg}$ of veratrine in the presence of naloxone (middle and bottom panels of Figure 2) were similar to those shown in Figure 1. Mean arterial blood pressures obtained immediately prior to the saline, naloxone alone, and $100 \mu \mathrm{g} / \mathrm{kg}$ of veratrine test trials were 107,108 , and $119 \mathrm{mmHg}$, respectively. An ANOVA of arterial blood pressures indicated a significant effect of drug $[\mathrm{F}(2,8)=5.06]$ and a significant effect of time $[F(4,16)=7.12]$, but no significant drug $\times$ time interaction. Mean baseline heart rates obtained immediately prior to the saline, naloxone alone, and $100 \mu \mathrm{g} / \mathrm{kg}$ of veratrine test trials were 478,516 , and $532 \mathrm{bpm}$. An ANOVA of heart rates indicated a significant effect of drug $[F(2,8)=27.46]$, a significant effect of time $[F(4,16)=9.45]$, and a significant drug $\times$ time interaction $[F(8,32)=3.34]$. However, analyses comparing the percentage changes in either arterial blood pressure or heart rate evoked by $100 \mu \mathrm{g} / \mathrm{kg}$ of veratrine in the absence or presence of naloxone indicated no significant differences.

In summary, Experiment 1 indicates that iv administration of veratrine is capable of inhibiting the tail-flick reflex evoked by painful radiant heat. Veratrine also elicits mild hypotension and strong bradycardia, which are characteristic of the Bezold-Jarisch reflex. The nociceptive and cardiovascular changes evoked by veratrine were unaffected by prior iv administration of naloxone. Moreover, the finding that magnitude of the cardiovascular responses to a $100-\mu \mathrm{g} / \mathrm{kg}$ dose of veratrine were not significantly different in either the presence or absence of naloxone suggests that the repeated veratrine drug administration procedure used on the first day of testing probably did not result in tachyphylaxis. If tachyphylaxis had occurred, it may have resulted in an underestimation of the actual antinociceptive action of $100 \mu \mathrm{g} / \mathrm{kg}$ of veratrine and a failure to detect an effect of naloxone on the second day of testing, when the repeated drug administration procedure was not used. Finally, it should be considered that, although large doses of veratrine were required to produce antinociceptive and cardiovascular changes in the rat, presumably only a small fraction of these doses of veratrine actually reach the coronary circulation from the systemic circulation to activate cardiac receptors, for example, left atrial $\mathrm{C}$-fibers in the rat, following the iv route of drug administration used in the present experiments.

\section{EXPERIMENT 2}

The antinociceptive actions of veratrine reported in $\mathrm{Ex}$ periment 1 may have been a consequence of activating cardiac receptors with associated vagal afferents. However, it is also possible that veratrine had either a direct action on the CNS or a nonspecific drug effect. The purpose of Experiment 2 was to determine the role of vagal afferents in mediating veratrine-induced antinociception by comparing the action of this drug in rats with right vagotomies, left vagotomies, or bilateral vagotomies. Specifically, if veratrine is operating to produce antinociception by activating vagal afferents, then either unilateral or bilateral resection of the vagus should attenuate or eliminate the antinociception.

\section{Method}

Subjects. Thirty-seven male Sprague-Dawley rats served as subjects. All other conditions were as described in Experiment 1.

Apparatus. The apparatus was as described in Experiment 1.

Surgical techniques. Since the rat cannot withstand bilateral vagotomy for any substantial period of time, the unilateral and bilateral resections were carried out under different experimental protocols with their own controls. In the unilateral condition, rats in the rightvagotomy $(\mathrm{N}=8)$, left-vagotomy $(\mathrm{N}=10)$, and sham-vagotomy $(\mathrm{N}=8)$ groups were anesthetized with ether and underwent the appropriate surgical treatment 4 weeks prior to testing. Rats in the right-vagotomy condition received a $3-\mathrm{cm}$ midventral incision in the cervical region. Dissection continued until the underlying sternocleidomastoid group was exposed. The right sternomastoid was displaced laterally and the deeper omohyoid was cut at right angles to its fiber direction. The right vagus was located and separated from the right common carotid artery and other surrounding tissues and nerves. The vagus was then cut and a $1-\mathrm{cm}$ segment removed inferior to the superior laryngeal nerve. Rats in the leftvagotomy condition received essentially the same surgical procedure, except that the left sternomastoid was displaced laterally and the left vagus was cut. Rats in the sham-vagotomy condition received the procedure described above for right vagotomy, but the vagus was not cut.

Four weeks later, these rats were instrumented with arterial and venous cannulae as described in Experiment 1. Twenty-four hours later, they were tested in the manner described in Experiment 1 with successive bolus iv administration of saline and 25,50 , and $100 \mu \mathrm{g} / \mathrm{kg}$ of veratrine. The $25-\mu \mathrm{g} / \mathrm{kg}$ dose was used in this experiment, since $5 \mu \mathrm{g} / \mathrm{kg}$ failed to produce an effect in the first experiment. All other aspects of the infusion procedure were as described in Experiment 1.

Rats in the bilateral vagotomy $(\mathrm{N}=6)$ and sham bilateral vagotomy $(\mathrm{N}=5)$ conditions were anesthetized with pentobarbital sodium $(50 \mathrm{mg} / \mathrm{kg})$ and received the implantation of the arterial and venous cannulae. Approximately 40-45 min following the administration of anesthesia, the vagi were resected in the bilateral vagotomy condition, whereas these nerves were merely exposed in the sham bilateral vagotomy condition. The rats were then immediately tested in the lightly anesthetized state using essentially the same procedure as was described in Experiment 1, with the following modifications. First, the rats were tested within 3 min following resection of the vagi, since this operation results in rapid death in the rat. Second, only the largest drug dose was administered $(100 \mu \mathrm{g} / \mathrm{kg})$. This was done to achieve the maximal antinociceptive effect, and preliminary work had indicated that repeated drug injections precipitated rapid cardiopulmonary failure in these animals.

\section{Results}

Mean tail-flick indices obtained following the administration of veratrine are presented in Figure 3 as a function of sham vagotomy, left vagotomy, and right vagotomy in the conscious rat preparation. Mean baseline tailflick latencies obtained prior to the initial test trials for the sham-vagotomy, left-vagotomy, and right-vagotomy 


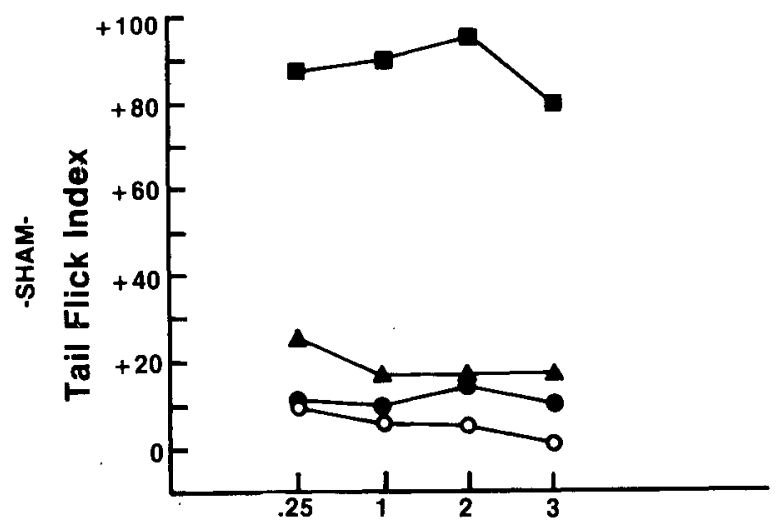

significantly from that of saline ( $\mathrm{Fs}=6.69$ and 110.99). However, no significant differences were found between the mean tail-flick indices produced by saline and the $25-\mu \mathrm{g} / \mathrm{kg}$ dose.

Figure 4 presents mean arterial blood pressures as a function of the percentage change from baseline values for the various groups. Mean baseline arterial blood pressures in the sham-vagotomy, left-vagotomy, and rightvagotomy groups were 136,126 , and $133 \mathrm{mmHg}$, respectively. In general, administration of veratrine results in
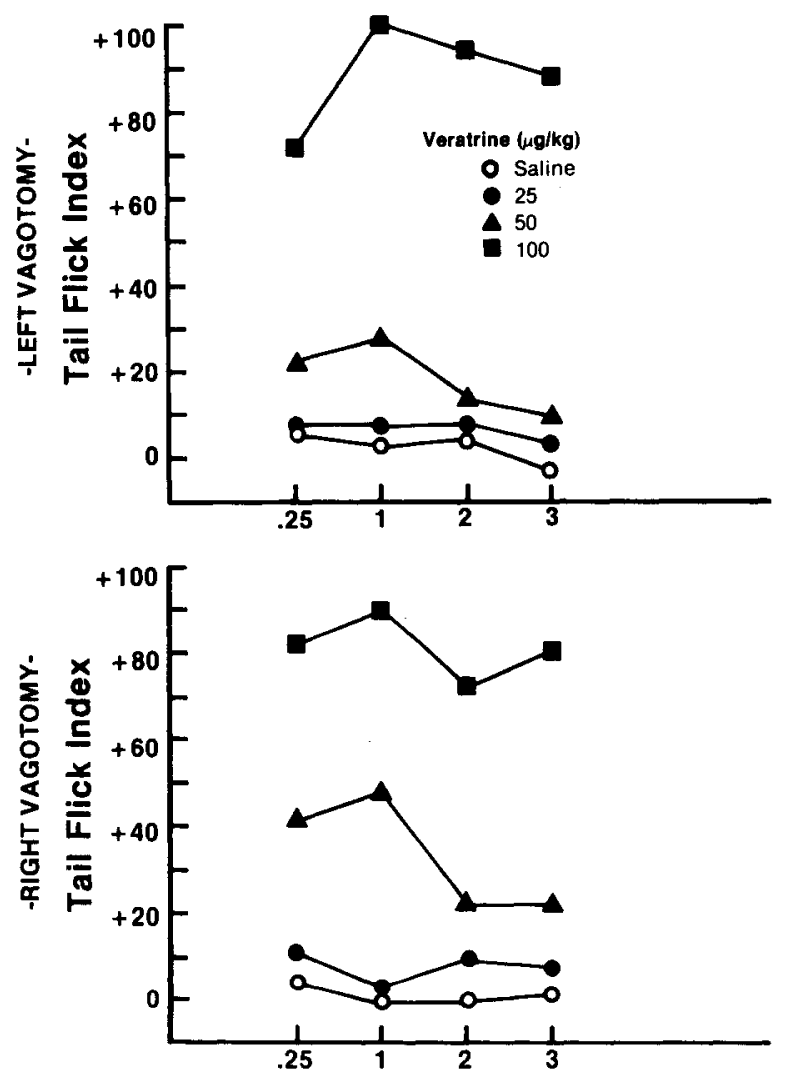

Minutes Post - Infusion

Figure 3. Mean tail-flick indices for the sham-vagotomy, leftvagotomy, and right-vagotomy groups following saline or veratrine administration.

groups were $3.69,4.18$, and $4.22 \mathrm{sec}$, respectively. In general, this figure reveals that there was no major effect of either a right or left vagotomy alone on tail-flick indices relative to sham vagotomy. This was confirmed by an ANOVA of tail-flick indices, which indicated no significant effect of operation $[\mathrm{F}(2,23)=0.17]$ and a significant effect of drug dose $[F(3,69)=138.69]$, but no significant interactions. Post hoc comparisons on the drug dose effect indicated that the mean tail-flick indices produced by both the $50-$ and the $100-\mu \mathrm{g} / \mathrm{kg}$ doses differed
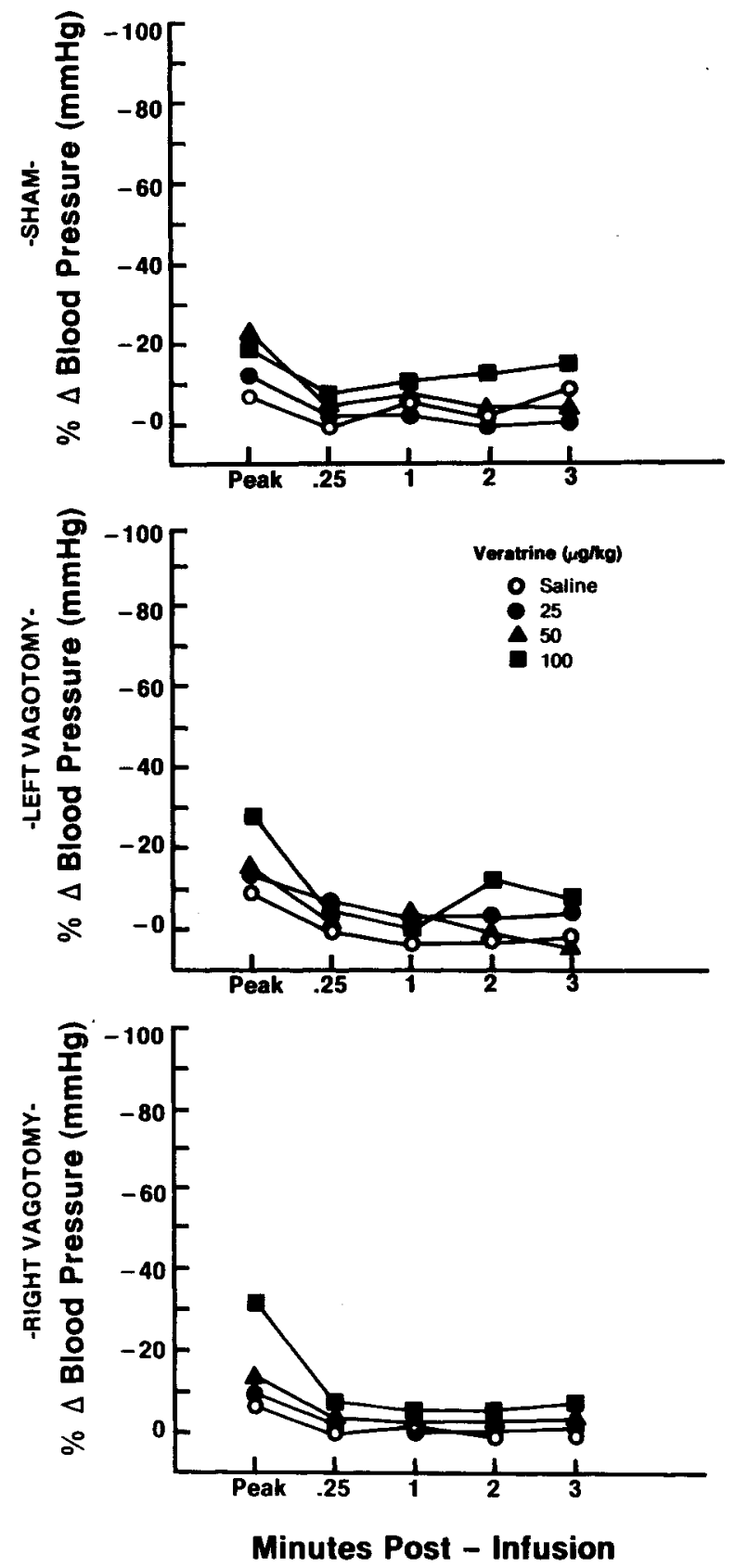

Figure 4. Mean arterial blood pressure expressed as percentage change from baseline for the sham-vagotomy, left-vagotomy, and right-vagotomy groups following saline or veratrine administration. 
modest hypotension, but analogous to the tail-flick data, there was little effect of either a right or left vagotomy on arterial blood pressure. This was confirmed by an ANOVA of arterial blood pressures indicating no significant effect of the operation $[F(2,23)=0.81]$, but significant effects of drug dose $[\mathrm{F}(3,69)=8.48]$ and time $[F(4,92)=34.81]$; none of the interactions were significant. Post hoc analysis means obtained at the peak time point indicated that the hypotension resulting from the $100-\mu \mathrm{g} / \mathrm{kg}$ dose differed significantly from that resulting from saline $(F=5.49)$. No other comparisons were significant.

Figure 5 presents the mean heart rates as a function of percentage change from baseline values for the groups. Mean baseline heart rates for the sham-vagotomy, leftvagotomy, and right-vagotomy groups were 500,516 , and $490 \mathrm{bpm}$, respectively. In general, this figure indicates that veratrine induces bradycardia, but that the magnitude of this effect was not substantially altered by either a right or left vagotomy. This view was supported by an ANOVA of heart rates, which indicated no significant effect of operation $[F(2,23)=0.19]$, but significant effects of drug dose $[F(3,69)=17.91]$ and time $[F(4,92)=51.37]$ and a significant effect of drug dose $\times$ time interaction $[F(12,276)=20.21]$. Post hoc analyses revealed that these differences were primarily due to the fact that the bradycardic responses in the $50-$ and $100-\mu \mathrm{g} / \mathrm{kg}$ doses at the peak time value were significantly larger than those evoked by saline (Fs $=4.52$ and 35.14, respectively).

In contrast to the failure of either right or left vagotomy alone to affect the antinociception produced by administration of veratrine, the top panel of Figure 6 reveals that bilateral vagotomy attenuates veratrine-induced antinociception in the anesthetized rat preparation. This was confirmed by two ANOVAs that indicated significantly greater tail-flick indices in sham-operated than in bilateral vagotomized rats $[\mathrm{F}(1,9)=24.52]$ and no significant differences in the magnitude of the antinociception manifested by the anesthetized sham-operated rats and the conscious sham-operated rats (compare the $100-\mu \mathrm{g} / \mathrm{kg}$ functions of Figure 3 and 6). Mean baseline tail-flick latencies in the sham-vagotomy and bilateral-vagotomy groups were 4.49 and $4.42 \mathrm{sec}$, respectively.

The middle panel of Figure 6 presents mean arterial blood pressures evoked by administration of veratrine expressed as percentage change from baseline values. Mean baseline arterial blood pressures in the sham-vagotomy and bilateral-vagotomy groups were 125 and $114 \mathrm{mmHg}$, respectively. An ANOVA of arterial blood pressures indicated no significant effect of operation $[F(1,9)=0.85]$, a significant effect of time $[F(4,36)=9.74]$, and a significant operation $\times$ time interaction $[F(4,36)=4.14]$. Follow-up comparisons revealed that bilaterally vagotomized rats showed significantly less hypotension than sham-operated rats only at the .25 time point $[F(1,9)=7.89]$.

Finally, mean heart rates are presented in the bottom panel of Figure 6, expressed as a function of percentage change from baseline. Mean baseline heart rates for the
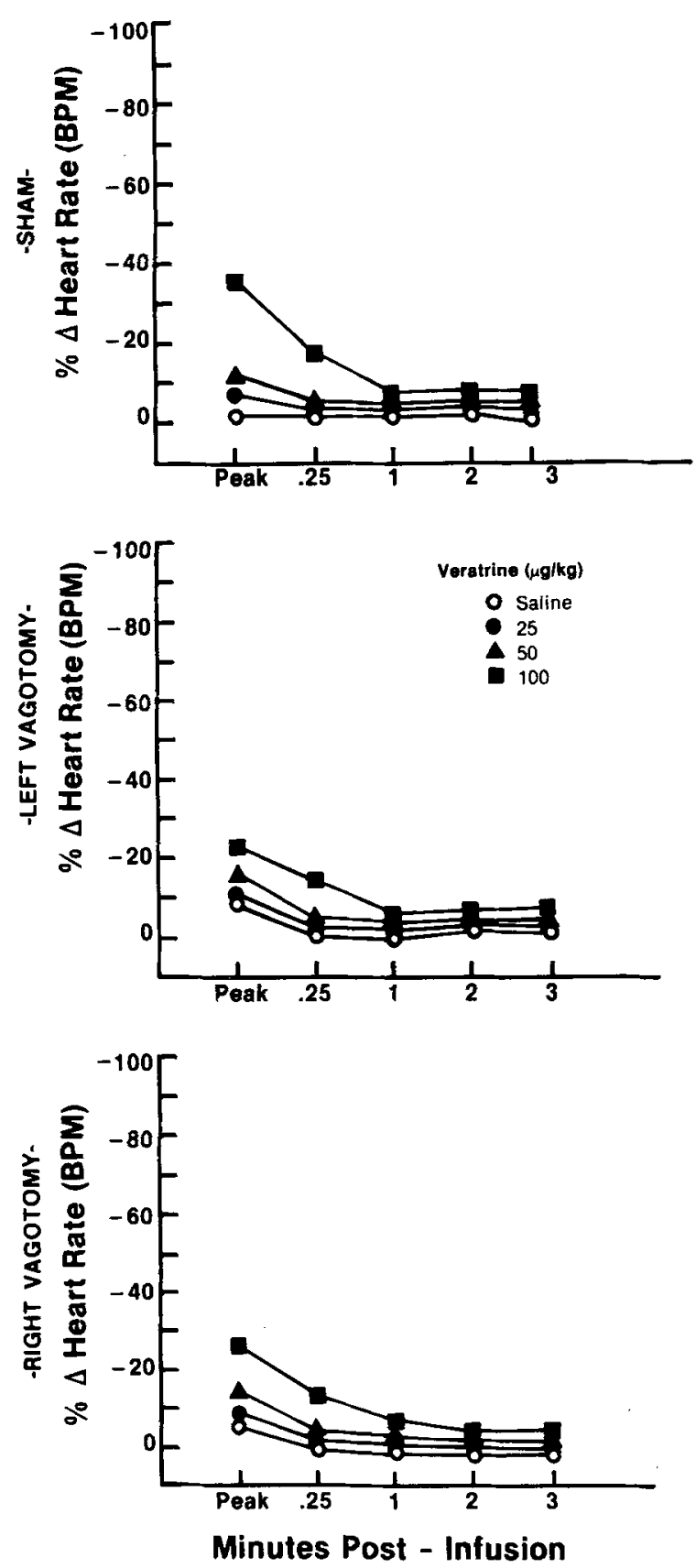

Figure 5. Mean heart rate expressed as percentage change from baseline for the sham-vagotomy, left-vagotomy, and right-vagotomy groups following saline or veratrine administration.

sham-vagotomy and bilateral-vagotomy groups were 446 and $427 \mathrm{bpm}$, respectively. An ANOVA of heart rates revealed no significant differences as a function of operation $[F(1,9)=2.07]$, but a significant effect of time was obtained $[F(4,36)=2.78]$. Clearly, the anesthetic resulted in a depression of cardiovascular reactivity, making interpretation of the heart rate data difficult.

In summary, Experiment 2 demonstrates that the antinociception produced by veratrine is a consequence of activation of vagal afferents. However, resection of either the right or left vagal nerve trunk does not attenuate the 

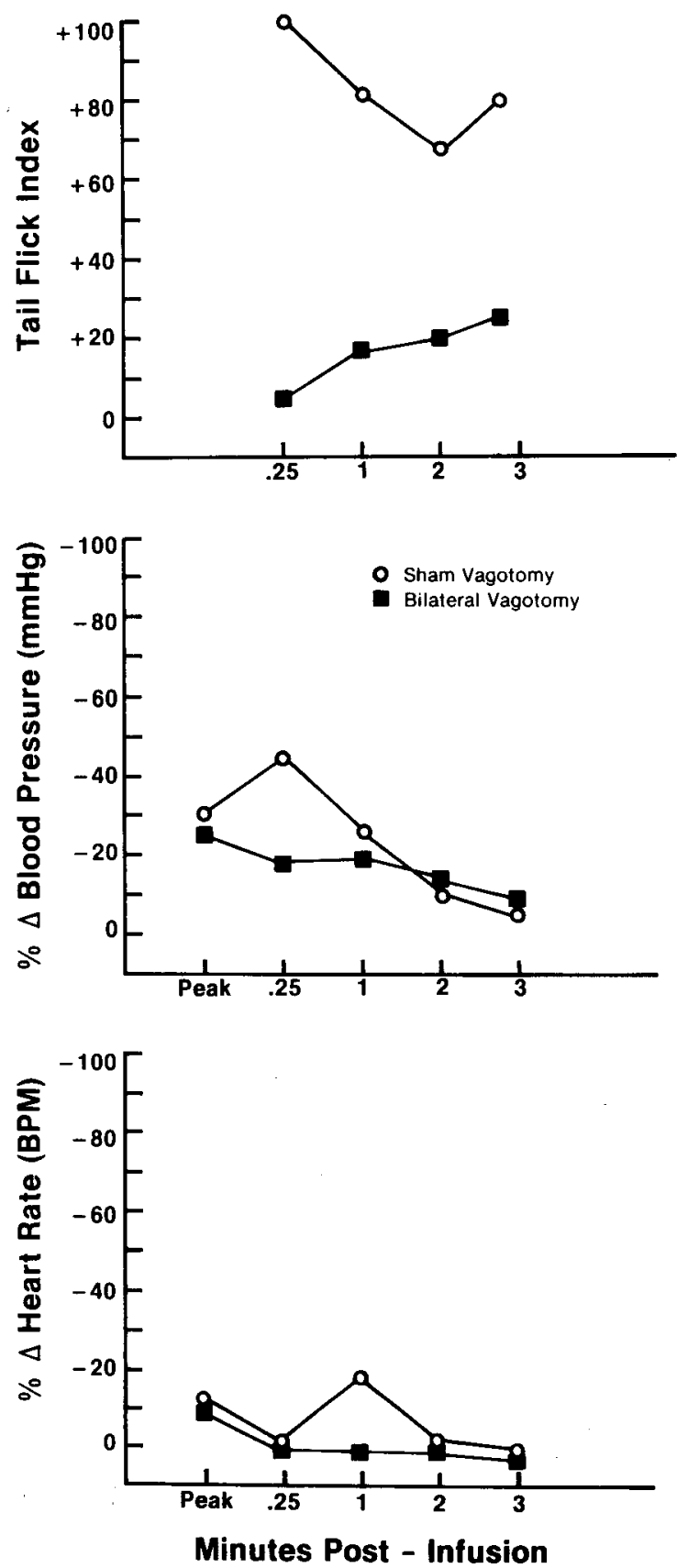

Figure 6. Mean tail-flick index, mean percent change in arterial blood pressure, and mean percent change in heart rate as a function of veratrine administration in bilateral vagotomized rats and sham controls.

antinociception. Specifically, both vagi must be resected to eliminate the antinociceptive effect of veratrine. As in Experiment 1, veratrine also resulted in reflex bradycardia and hypotension.

\section{EXPERIMENT 3}

Experiments 1 and 2 indicate that veratrine activates vagal afferents to produce inhibition of the tail-flick reflex evoked by painful radiant heat. This antinociceptive action of veratrine was unaffected by pretreatment with naloxone, thereby suggesting the possibility of mediation by a nonopioid pain-inhibition system. The purpose of Experiment 3 was to determine if iv administration of veratrine would also inhibit the paw-lick response evoked by painful thermal stimulation in the hot-plate assay. In this manner, it is possible to more fully characterize the range of response systems affected by veratrine. The efficacy of naloxone in affecting antinociception induced by veratrine in the hot-plate assay was also examined.

\section{Method}

Subjects. Thirty-two male Sprague-Dawley rats served as subjects. All other conditions were as described in Experiment 1.

Apparatus. The hot-plate apparatus consisted of a circular copper plate $(20 \mathrm{~cm}$ in diameter) immersed in a water bath. The temperature of the plate was maintained at $51^{\circ} \pm .05^{\circ} \mathrm{C}$ by a constant temperature control unit. The copper plate was enclosed by a $45-$ $\mathrm{cm}$ Plexiglas cylinder, which prevented escape from the hot-plate.

Surgical techniques. All rats received implantation of only a venous cannula in the external jugular vein, as described in Experiment 1 .

Testing. Twenty-four hours later, all rats received testing in the hot-plate assay of pain sensitivity. On the first test day, four groups of rats $(\mathrm{N}=8 /$ group $)$ received a bolus iv infusion of saline or 25 , 50 , or $100 \mu \mathrm{g} / \mathrm{kg}$ of veratrine while restrained. Each drug administration was followed by a $400-\mu \mathrm{l}$ saline flush. Fifteen seconds later, each rat was placed on the hot plate, and the latency to either lick a hind paw or jump from the plate was recorded with a stopwatch. Failure to respond within $90 \mathrm{sec}$ resulted in removal from the hot plate to prevent tissue damage to the paws.

On the following day, the same experimental protocol was used, except that all rats received an ip injection of $20 \mathrm{mg} / \mathrm{kg}$ of naloxone $\mathrm{HCl}$ approximately $10-15 \mathrm{~min}$ prior to an iv infusion of veratrine (the same dose as on the preceding day) and testing on the hot-plate. Thus, this design permitted a between-groups comparison of veratrine-induced antinociception in the hot-plate assay and a within-group comparison of the efficacy of naloxone in affecting this antinociception.

\section{Results}

Figure 7 presents group mean hot-plate response latencies. This figure indicates that latencies to paw-lick systematically increased as a function of the dose of veratrine. An ANOVA of response latencies indicated a significant between-groups effect $[\mathrm{F}(3,28)=5.92]$. However, post hoc comparisons indicated that the mean response latency of only the $100-\mu \mathrm{g} / \mathrm{kg}$ group differed significantly from that of the saline control $(\mathrm{F}=5.53)$. It is our impression, however, that the failure to observe significant antinociceptive actions in the $25-$ and $50-\mu \mathrm{g} / \mathrm{kg}$ groups was a consequence of variability created by the testing procedure itself, that is, physical restraint followed by a bolus infusion. Specifically, it is likely that these doses would result in inhibition of the paw-lick response if all groups were adapted to the infusion procedure prior to testing. It is also useful to note that virtually no animal showed any signs of motor impairment which could have precluded responding in either the hot-plate or tail-flick assays. Pretreatment with $20 \mathrm{mg} / \mathrm{kg}$ of systemic naloxone did not affect the antinociception induced by iv veratrine on the second day of testing $(F<1)$. 


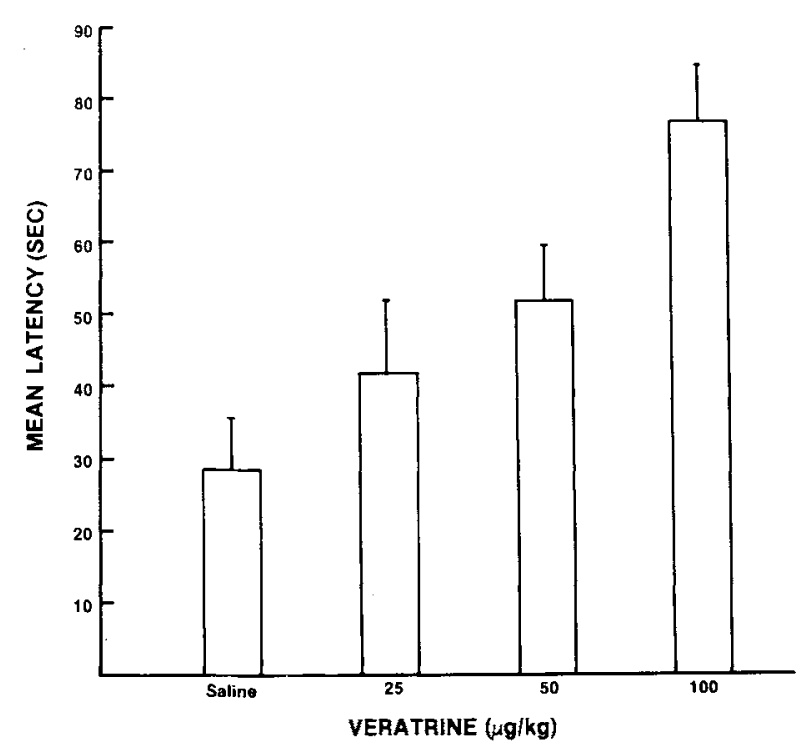

Figure 7. Mean paw-lick response latencies following saline or veratrine administration.

Thus, the present study extends the range of painful stimuli and response systems that are affected by veratrine to thermal stimuli and the paw-lick response.

\section{GENERAL DISCUSSION}

Randich and Maixner (1984b) proposed the view that systems involved in cardiovascular regulation are physiologically linked to systems controlling the perception of pain. Specifically, their view suggests that either peripheral or central activation of the sinoaortic and/or the cardiopulmonary baroreceptor reflex arc results in antinociception. This assertion has been supported by several lines of evidence. First, activation of the sinoaortic baroreceptor reflex arc by either a continuous or bolus infusion of phenylephrine (a peripheral vasoconstrictor) results in inhibition of the tail-flick reflex to radiant heat, and this antinociception cannot be induced in rats with bilateral sinoaortic deafferentation or SAD (Randich \& Hartunian, 1983; Randich \& Maixner, 1984b). A bolus infusion of phenylephrine also produces a profound antinociceptive effect in rats tested in the hot-plate assay of pain sensitivity (Randich, unpublished observations). Conversely, bilateral SAD has been shown to produce hyperalgesia to electric shock stimuli in SHRs and Wistar-Kyoto normotensive (WKY) rats (Randich, 1982). Second, activation of the cardiopulmonary baroreceptor reflex arc by volume expansion also results in inhibition of the tailflick reflex, and this effect is attenuated by right vagotomy (Maixner \& Randich, 1984). Third, activation of peripheral opioid receptors with associated vagal afferents by administration of DALA results in inhibition of the tail-flick reflex. This antinociceptive action of DALA is eliminated by either pretreatment with naloxone or bilateral vagotomy, but is unaffected by unilateral right vagotomy or bilateral SAD (Randich \& Maixner, 1984a). Finally, resection of the right vagal nerve trunk has been shown to produce both a relative hyperalgesia in SHRs and WKYs (Maixner et al., 1982) and an attenuation of SIA (Maixner \& Randich, 1984).

The present experiments also support this view by showing that veratrine-induced antinociception is a consequence of peripheral activation of vagal afferents. It is similar to the antinociception produced by volume expansion in that opioid receptor antagonists are without effect, that is, within the dosage levels and types of antagonists tested. However, veratrine- and volume expansioninduced antinociception differ in that unilateral resection of the right vagal nerve trunk has no effect on the former, but does attenuate the latter. This suggests that the mechanoreceptors activated by volume-expansion represent either a different population of receptors from those activated by veratrine or a subpopulation of those receptors that are activated by veratrine, although both inhibit pain via vagal input to the CNS. In contrast, veratrine and DALA-induced antinociception are similar to each other in that both are eliminated following bilateral, but not unilateral, vagotomy. They differ, however, in that pretreatment with naloxone eliminates antinociception resulting from DALA but not veratrine administration. These important differences are not surprising given the lack of information about opioid effects on cardiac receptors (Barron \& Bishop, 1982; Thoren, 1979; Walker, Thames, Abboud, Mark, \& Klopfenstein, 1978).

Experiment 3 also showed that veratrine induces antinociception in the hot-plate assay of pain sensitivity, and informal observations indicated that there was little or no motor impairment. These findings not only extend the response systems that are affected by the proposed cardiovascular-pain regulatory interactions (Randich \& Maixner, 1984b), but also suggest that a true analgesic effect may be involved.

Finally, the hypotensive and bradycardic effects of veratrine observed in the present studies are consistent with information previously established about the actions of veratrum alkaloids on cardiac receptors (Barron \& Bishop, 1982; Thoren, 1979; Walker et al., 1978). We assume that these cardiovascular responses are mediated by cardiopulmonary receptors with associated vagal afferents, because they occurred with 1-2 sec of drug infusion, but no definitive statement can be made on the basis of the present data, since cardiovascular responsivity was markedly depressed in both the sham-vagotomy and bilateral-vagotomy anesthetized rat preparations. However, some hypotension was observed in bilaterally vagotomized rats following administration of veratrine in the absence of any reflex bradycardia, suggesting that at least some of the hypotensive actions of this drug are not vagally mediated. Although veratrine will activate sympathetic afferents arising from the heart, the reflex adjustment to cardiac sympathetic activation is typically excitatory, not inhibitory, in nature (Bishop et al., 1984). It is of interest, however, that the cardiorespiratory 
responses evoked by veratrine are very similar to those evoked by various met- and leu-enkephalinamide analogues (Randich \& Maixner, 1984a; Willette \& Sapru, $1982 \mathrm{a}, 1982 \mathrm{~b})$. It is possible, therefore, that veratrine produces at least some of these cardiovascular effects by increasing ongoing activity of vagal afferents with associated cardiopulmonary opioid receptors.

\section{REFERENCES}

Ammons, W. S., Blair, R. W., \& Foreman, R. D. (1983a). Vagal afferent inhibition of primate thoracic spinothalamic neurons. Journal of Neurophysiology, 50, 926-940.

Ammons, W. S., Blair, R. W., \& Foreman, R. D. (1983b). Vagal afferent inhibition of spinothalamic cell responses to sympathetic afferents and bradykinin in the monkey. Circulation Research, 53, 603-612.

BARRON, K. W., \& Bishop, V. S. (1982). Reflex cardiovascular changes with veratridine in the conscious dog. American Journal of Physiology, 242, H810-H817.

Bishop, V. S., Malliani, A., \& Thoren, P. (1984). Cardiac mechanoreceptors. In J. T. Shepherd \& F. M. Abboud (Eds.), Handbook of physiology - The cardiovascular system III. (pp. 497-555). Bethesda, MD: American Physiological Society.

KuPChAN, S. M., \& FlaCKE, W. E. (1967). Hypotensive veratrum alkaloids. In E. Schlittler (Ed.), Antihypertensive agents. (pp. 429458). New York: Academic Press.

MAIXNER, W., \& RANDICH, A. (1984). Role of the right vagal nerve trunk in antinociception. Brain Research, 298, 374-377.

Maixner, W., Touw, K. B., Brody, M. J., Gebhart, G. F., \& Long, J. P. (1982). Factors regulating the altered pain perception in the spontaneously hypertensive rat. Brain Research, 237, 137-145.
RANDICH, A. (1982). Sinoaortic baroreceptor reflex arc modulation of nociception in spontaneously hypertensive and normotensive rats. Physiological Psychology, 10, 267-272.

Randich, A., \& HaRTunian, C. (1983). Activation of the sinoaortic baroreceptor reflex arc induces analgesia: Interactions between cardiovascular and endogenous pain inhibition systems. Physiological Psychology, 11, 214-220.

RANDich, A., \& MAIXNeR, W. (1984a), [D-Ala $\left.{ }^{2}\right]-$ methionine enkephalinamide reflexively induces antinociception by activating vagal afferents. Pharmacology, Biochemistry, and Behavior, 21, 441-448.

RANDICH, A., \& MAIXNER, W. (1984b). Interactions between cardiovascular and pain regulatory systems. Neurosciences and Biobehavioral Reviews, 8, 343-367.

TheIs, R., \& Foreman, R. D. (1981). Descending inhibition of spinal neurons in the cardiopulmonary region by electrical stimulation of vagal afferent nerves. Brain Research, 207, 178-183.

THOREN, P. (1979). Role of cardiac vagal C-fibers in cardiovascular control. Review of Physiology, Biochemistry, and Pharmacology, 86, $1-94$.

Walker, J. L, Thames, M. D., Abboud, F. M., Mark, A. L., \& KlopFENSTEIN, H. S. (1978). Preferential distribution of inhibitory cardiac receptors in the left ventricle of the dog. American Journal of Physiology, 235, H188-H192.

Willette, R. N., \& SAPRU, H. N. (1982a). Peripheral versus central cardiorespiratory effects of morphine. Neuropharmacology, 21, 1019-1026.

Willette, R. N., \& SAPRU, H. N. (1982b). Pulmonary opiate receptor activation evokes a cardiorespiratory reflex. European Journal of Pharmacology, 78, 61-70.

(Manuscript received July 5, 1984; revision accepted for publication December 20, 1984.) 